\title{
Parametric ordering of complex systems
}

\author{
P.-M. Binder \\ IIASA, A-2361 Laxenburg, Austria \\ and \\ Wolfson College, Oxford OX2 6UD, United Kingdom
}

\begin{abstract}
Cellular automata (CA) dynamics are ordered in terms of two global parameters, computable a priori from the description of rules. While one of them (activity) has been used before, the second one is new; it estimates the average sensitivity of rules to small configurational changes. For two well-known families of rules, the Wolfram complexity Classes cluster satisfactorily. The observed simultaneous occurrence of sharp and smooth transitions from ordered to disordered dynamics in CA can be explained with the two-parameter diagram.
\end{abstract}

PACS numbers: $05.45 .+\mathrm{b}, 02.60 .-\mathrm{x}, 05.70 .-\mathrm{a}, 89.80 .+\mathrm{h}$ 
In recent years cellular automata $(\mathrm{CA})$ have played two important roles. As totally discrete models (in space, time and state variable) they have proved very useful for the computer simulation of diverse spatially extended problems in physics, chemistry and biology ${ }^{1-6}$. They have also been an important tool in the classification of complex spatio-temporal behavior ${ }^{7}$ and in the understanding of the origins of complexity in physical systems ${ }^{8}$. In this paper we address the related problem of ordering CA rules in terms of parameters that can be calculated a priori from the description of a rule, without performing simulations. We do this in terms of the well-studied activity parameter ${ }^{9}$, described below, and of a new parameter which estimates average sensitivity to small changes in configuration. The two main results are that rules cluster satisfactorily in the two-parameter diagram according

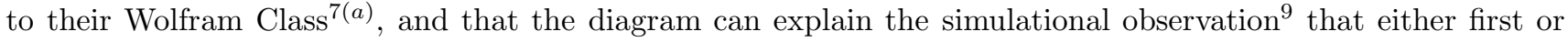
second-order transitions from ordered to disordered dynamics can be observed over the same range of the activity parameter.

This work concentrates on one-dimensional, two-state $(k=2)$ totalistic rules with radius $r=2$. For these, sites which can take on the values zero or one are updated simultaneously at integer time steps based on deterministic functions $f$ of the sum of the five neighbors. Upon defining zero as a quiescent state (the outcome of the neighborhood 00000 is 0$), 32$ rules remain in this family. Results for elementary CA $(k=2, r=1)$ will also be sketched here, and will be presented in detail elsewhere ${ }^{10}$. These two families have been chosen because simulations of their behavior are quite well documented (Ref. 3). The four Wolfram Classes are represented in this set of rules. They are: (1) evolution leading to a homogeneous state (all zeros or ones); (2) evolution leading to a set of separated simple or periodic structures; (3) evolution leading to chaotic patterns, and (4) evolution leading to complex localized structures, sometimes long-lived. Examples of these classes can be found in Ref. 3 . Classes 1 and 2 are considered to be ordered, Class 3 is chaotic, and Class 4 is somewhere in between, exhibiting complex structures that live for very long times ${ }^{11}$.

Langton and coworkers ${ }^{9}$ identified a global parameter that turns out to be a useful predictor of the complexity of a $\mathrm{CA}$ rule. Their activity parameter $\lambda$ is the fraction of non-zero outputs of the CA transition function $f$ when all possible arguments (neighborhoods) of $f$ are weighted equally. The activity parameter 
$(0 \leq \lambda \leq 1)$ estimates roughly the asymptotic fraction of active (non-zero) sites. Clearly, rules where most sites go to zero or to one very quickly tend to homogeneous global states (Class 1), while on the other hand at intermediate values of activity there are more global states to be explored and one would expect more disorder ${ }^{12}$. In Refs. 9, several effectively continuous measures of complexity ${ }^{13}$ were monitored as $\lambda$ was increased. A correlation between complexity and activity was found, but it has become clear that this parameter alone cannot predict the dynamics of a rule. For example, the identity rule, which freezes in time all initial conditions, has $\lambda=1 / 2$ and yet is quite ordered. When the number of states or neighborhood size is large enough that $\lambda$ is suitably fine-grained, the order-to-chaos transition with increasing activity sometimes happens gradually (reminiscent of a second-order transition, usually via Class 4 rules), and other times suddenly, as in a first-order phase transition ${ }^{9}$. This points to the need for additional parameters. Attempts have been made by defining generalized versions of thermodynamic quantities or quantities derivable from mean-field theories ${ }^{9}$, but no satisfactory answers have emerged yet.

To address this need we have used a new sensitivity parameter $(\mu)$, motivated by the numerical observation that the Wolfram Classes are not only characterized by their spatio-temporal patterns, but also by their sensitivity to changes of the values of one or a few sites (see Ref. $7(\mathrm{a})$ for example). We therefore define the sensitivity parameter as the fraction of changes in the evolution caused by changing the value of a site, averaged over all sites of all neighborhoods in the rule table:

$$
\mu=\frac{1}{n m} \sum_{n} \sum_{j=1}^{m} \frac{\partial f}{\partial s_{j}}
$$

where $n$ are the possible neighborhoods in the rule table, and $m$ is the number of bits in the neighborhood. The Boolean derivative for $\mathrm{CA}^{14}$ is $\partial f / \partial s_{j}=1$ if $f\left(s_{1}, \ldots, s_{j}, \ldots\right) \neq f\left(s_{1}, \ldots,-s_{j}, \ldots\right)$, this is, if the value of $f$ is sensitive to the value of the bit $s_{j}$, and zero otherwise. The minus sign is taken to mean bit complementation. This estimates the average sensitivity to changes in a rule in the same spirit as $\lambda$ estimates the asymptotic concentration of active sites, and is in some sense analogous to a spatial Lyapounov exponent. A crucial point of this work is that average sensitivity can be estimated a priori and used as a predictor of complexity, instead of measured from simulations and used as a measure of complexity as has been done in Ref. 15 for the average 
difference pattern spreading rate.

A two-parameter diagram of totalistic $(k=2, r=2)$ rules is shown in Figure 1. It is seen that (1) all Class 1 rules cluster at low $(\lambda, \mu),(2)$ Class 2 rules are contained in two separate clusters, at intermediate $(\lambda, \mu)$, (3) Class 4 rules occupy a high-sensitivity, medium-activity region and (4) Class 3 rules are quite prevalent and occupy much of the parameter space. The clustering of rules by Wolfram Class into distinct portions of rule space is satisfactory, but several comments are in order. First, the line between ordered rules and chaotic rules is sometimes as sharp as the coarse-graining, $\Delta \lambda=1 / 32$, allows; this indeed suggests possible first-order dynamical phase transitions as activity is increased. Second, the isolated bubble of Class 2 rules at higher activity was expected $^{9}$, and corresponds in this case to rules in which neighborhoods of sum 3 and 4 (and 5) produce ones at the next time step. The resulting frozen patterns of thick bands of ones and zeros are typical of Class 2. Third, we have folded the diagram along the line $\lambda=1 / 2$, as low and high activity are dynamically equivalent; the rules for $\lambda>1 / 2$ are shown in empty rather than full symbols. Fourth, the arrows A and B demonstrate how, for the same value of activity, one can observe either sharper or smoother transitions depending on the sensitivity parameters of the rules. Fifth, as the sensitivity parameter increases with $\lambda=1 / 2$ fixed, we see the expected sequence of Classes (1-2, 4 and 3). Sixth, the Class 3 rule with very high activity and low sensitivity which folds near the bottom left corner of the diagram is atypical ${ }^{16}$. Seventh, the subclass of totalistic, quiescent rules may not be indicative of the larger space of $2^{32}(k=2, r=2)$ rules because of special symmetries. Finally, the activity-sensitivity diagram for the 88 independent elementary CA looks quite similar, but it only has three convex domains for Classes 1, 2 and 3 .

The results in this paper help us understand the space of CA rules, which can exhibit spatio-temporal patterns ranging from ordered to complex to chaotic. In particular, we have proposed a new parameter, which estimates a priori the average sensitivity of rules to small perturbations. The resulting diagrams in parameter space show that the rules cluster according to their Wolfram Classes. In fact, $\mu$ alone appears to be a better predictor of complexity than $\lambda$. There are exceptions, such as the Class 2 pocket with higher $\lambda$, $\mu$, which remind us that such simple parameters do not capture all the subtleties of rule dynamics. Exceptions probably exist even 
with larger $k, r$, and dimension. The diagrams also illustrate how different types of dynamical phase transitions can occur over the same range of the activity parameter. It is encouraging that the two parameters work well in low-dimensional, small-radius rules, where correlations are known to be the worst; we are currently investigating the rule space of higher-dimensional CA and hope that this work will be extended to study the relation between the parameters presented here and those used to describe continuous-variable complex systems such as coupled-map lattices.

Two final remarks are in order. One is that $\mu$ may be refined by separating the sensitivity to the central and peripheral sites (corresponding roughly to information generation and transmission); this is currently being investigated. The second remark is that ordering in terms of such simple parameters is heuristic rather than rigorous: the use of the activity parameter has recently been criticized ${ }^{17}$. However, careful examination of the rules that escape simple predictions can lead to increasingly sharp definitions of difficult concepts such as complexity.

The author thanks the French Association for the Development of Systems Analysis for financial support, and R. Bagley, C.G. Langton, W. Li, M. Mitchell, K. Sigmund, and A. Wuensche for suggestions. 


\section{References}

[1] J.D. Farmer, T. Toffoli and S. Wolfram, eds. Cellular automata (North-Holland, Amsterdam, 1984).

[2] J. Demongeot, E. Goles and M. Tchuente, eds., Dynamical systems and cellular automata (Academic, New York, 1985).

[3] S. Wolfram, ed., Theory and applications of cellular automata (World Scientific, Singapore, 1986)

[4] P. Manneville et al., eds., Cellular automata and the modeling of complex physical systems (Springer, Berlin, 1989)

[5] H. Gutowitz, ed., Cellular automata: Theory and experiment (North-Holland, Amsterdam, 1990)

[6] G.D. Doolen et al. eds., Lattice gas methods for partial differential equations (Addison-Wesley, New York, 1990)

[7] (a) S. Wolfram, Physica D 10, 1 (1984); (b) W. Li and N. H. Packard, Compl. systems 4, 281 (1990), K. Sutner, Physica D 45, 386 (1990), H. A. Gutowitz, ibid. 45, 136 (1990), P.-M. Binder, J. Phys. A: Math. Gen. 24, L21 (1991); ibid. 241677 (1991).

[8] S. Wolfram, Phys. Rev. Lett. 55, 449 (1985); P. Grassberger, Helv. Phys. Acta 62, 489 (1989).

[9] C. G. Langton, Physica D 22, 120 (1986), ibid. 42, 12 (1990); W. Li, N. H. Packard, and C. G. Langton, ibid. 45, 77 (1990), W. K. Wootters and C. G. Langton, ibid. 45, 95 (1990).

[10] P.-M. Binder (unpublished).

[11] We have chosen the Wolfram classification because it is the most prevalent in the literature. We note that it has been refined by Li and Packard (Ref. 7), that it has been argued that in certain cases assigning a Class to a rule is undecidable in infinite lattices: K. Culik II and S. Yu, Compl. systems 2, 177 (1988), and that sometimes the underlying patterns are not obvious to eye inspection: Ref. 3, and N. Boccara, J. Nasser, and M. Roger, Phys. Rev. A 44, 866 (1991). 
[12] This argument neglects matters of broken ergodicity and inaccessibility of states.

[13] For example, site or block entropies and mutual information are particularly useful measures. Both are used in Ref. 9.

[14] G. Y. Vichniac, Physica D 45, 63 (1990).

[15] N. H. Packard, in J. A. S. Kelso, A. J. Mandell and M. F. Shlesinger, eds. Dynamic patterns in complex systems (World Scientific, Singapore, 1988), pp. 293-301.

[16] This rule has a single-humped mean-field return map; such rules often tend to be chaotic, as discussed by Li and Packard (Ref. 7), H. V. McIntosh, Physica D 45, 105 (1990), and W. Li, J. Stat. Phys. 68, 829 (1992).

[17] M. Mitchell, P. T. Hraber, and J. P. Crutchfield, Compl. systems 7, 89 (1993). 
Figure 1. Phase diagram of rules for totalistic $(k=2, r=2)$ cellular automata. Horizontal axis: activity parameter. Vertical axis: sensitivity parameter. Circles: ordered rules (Wolfram Classes 1 and 2, with Class 2 rules encircled and labeled C2); Squares: complex rules (Wolfram class 4); Triangles: disordered or chaotic rules (Wolfram class 3). Open symbols correspond to rules with large $\lambda$, which have been reflected about the $\lambda=1 / 2$ axis. The arrows correspond to a first-order phase transition (A), and to a second-order phase transition (B). Any symbol may correspond to more than one rule. 